\title{
Highly-Integrated Power Cell for High-Power Wide Band-gap Power Converters
}

\author{
Jordi Espina, Behzad Ahmadi, Lee Empringham, Liliana De Lillo, Mark Johnson \\ Power Electronic, Machines and Control group \\ University of Nottingham \\ Nottingham, United Kingdom \\ mark.johnson@nottingham.ac.uk
}

\begin{abstract}
The fast switching speeds and low specific conduction losses of wide band-gap semiconductors allow the realisation of high-frequency, high power-density switching converters with dramatically reduced passive component requirements compared to Silicon technology. However, careful attention must be paid to switching cell design to mitigate the effects of circuit parasitics and fast voltage transitions which would otherwise limit the attainable switching speed and lead to increased levels of EMI. This paper presents a modular, powercell solution which allows the creation of any two-level topology converter. The cell structure enables fast switching of wide bandgap semiconductor devices while allowing high power converters to be fabricated using multiple, smaller commutation cells. Close integration of semiconductor dies, decoupling capacitors, gate drives and an output filter with a single ceramic substrate to act as the thermal path allows dramatic increases in power density without compromising converter performance. ${ }^{1}$
\end{abstract}

Keywords-Power electronics integration; wide bandgap semiconductors; power-density

\section{INTRODUCTION}

One of the main driving forces in power electronics development is that of increasing the power density of power converters $[1,2]$. This tendency is due to different reasons, reduced space, raw material cost, ability to integrate power converters inside machines and performance increases. This subject has also gained momentum with the development of new wide bandgap semiconductor devices such as those utilising Silicon Carbide or Gallium Nitride, together with the advantages that they bring, such as high switching speed, higher temperature operation, superior thermal conductivity and robustness. This paper introduces a modular strategy which allows the reduction of the overall size of the converter and a considerable increase in power density. Due to its modular approach, it also enables the creation of any multiphase converter, which permits connection to multi-phase motors

${ }^{1}$ This work was supported by the Engineering and Physical Sciences Research Council (EPSRC) National Centre for Power Electronics under grant reference EP/K035304/1. which are presently finding more and more research applications [3, 4].

\section{Single BASIC POWER CELl}

The most common topology used to create a Voltage Source Inverter (VSI) within the industrial drives industry is the two-level inverter. This topology is shown in Fig.1 where it may be seen that it is formed using two uni-polar active switching devices connected in series and one diode connected in parallel with each active switch. This simple circuit allows the designer to create any two level converter configuration with different numbers of $n$ output phases.

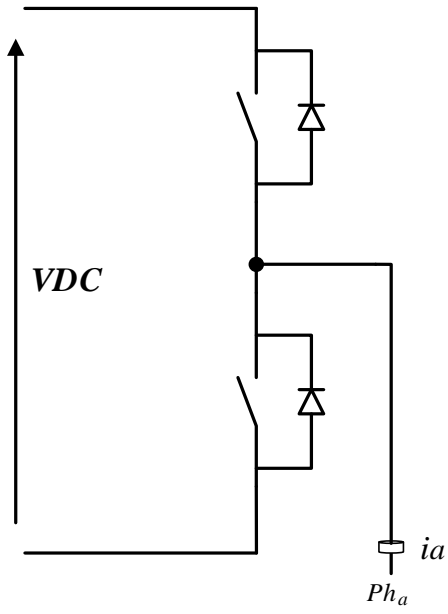

Fig. 1. Example Single two level VSI leg

The methodology used by semiconductor power module manufacturers at present to create high power drives is that, as ever increasing current ratings are needed, more and more individual semiconductor devices are placed and wired in parallel within the power module. As the physical size of the substrate used to mount the power devices increases, the parasitic inductances within the power module also increase. This inevitably means that the switching speed of the devices within the power module has to be reduced in order to minimize the damaging effects of the increased parasitic 
inductance. Also, different electromagnetic paths for the current through different devices means that all of the parallel devices which make up one 'switch' do not dynamically share the load current correctly and as a result, their switching speed needs to be reduced further.

This study uses the basic topology shown in Fig.1 in order to create a modular circuit cell using wide bandgap switching devices. This modular circuit has the flexibility to create any multiphase converter independent of the number of phases and output current. Higher current outputs can be achieved by simply paralleling the modular cell. Throughout this paper, this modular circuit will be referred as Standard Basic Power Cell (SBPC). As mention before, this SBPC is formed using two switches and two diodes. It also includes the necessary passive components to permit the interconnection between SBPCs. The output waveform is also filtered at source. The circuit proposed is showed in Fig.2.

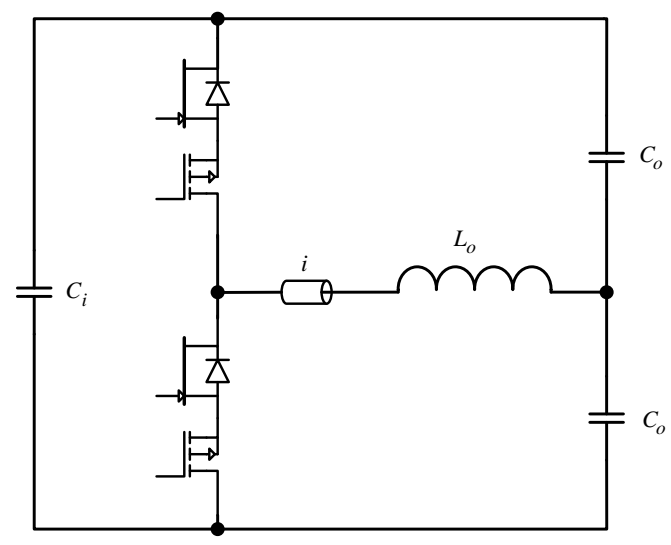

Fig. 2. Single Basic Power Cell circuit

The main driving force for this development, given the chosen circuit topology, was to create an optimized commutation circuit with a suitable innovative layout to minimize the commutation loop inductance. This is further facilitated with the inclusion of the DC port capacitance $\mathrm{Ci}$, on the substrate. This allows the semiconductor devices to switch at their maximum speed with minimal voltage overshoot at the output port. In order to facilitate the paralleling of these switching cells to form higher power converters, inductors are included at the output port. In order to filter the output waveform and reduce Electro-Magnetic Interference (EMI) generated by the switching cells, output capacitances are also included.

To create a commutation cell with minimal parasitic inductance, it is desirable to integrate the passive components at the substrate level. This creates the situation where the EMI could be contained at the source of the EMI and not be allowed to propagate around the converter to the outside world. In turn, this reduces the need for separate EMI filters at the input and output ports of the converter. The key to the integration of the passive components inside the SBPC is to reduce their physical size. One of the solutions to achieving this is reducing their nominal values, however this solution involves an inevitable increase in the current and voltage ripples. This methodology would be acceptable if there were a substantial increase in the switching frequency of the switches. Typical commercial VSI's use Si IGBTs which are switched at frequencies of between $1 \mathrm{kHz}$ and $20 \mathrm{kHz}$ being limited by the inherent capability of these semiconductors. In order to overcome this physical limitation a change in the semiconductor switch technology was mandatory. This study used SiC JFETS to achieve higher switching frequencies, although SiC MOSFETs or GaN HEMTs could also be applied. These transistors can switch at least one order of magnitude faster than traditional silicon solutions for the desired voltage capability of over 600V. Fig. 2 shows the JFETs together with P-MOS Silicon MOSFETS, in a cascode configuration, to create normally ' $O F F$ ' switching devices. Note that the P-MOS devices are only actively switched during power-up of the converter, the JFETs being driven directly during normal operation.

Following the structure proposed and using the semiconductors mentioned above a SBPC was designed in order to accomplish the maximum power whilst utilizing the minimum space. The requirements were $240 \mathrm{~V}$ (RMS) @ $50 \mathrm{~A}$ (RMS) output while keeping the THD of the output voltage and current to a minimum. This step of integration increases substantially the power density of a typical high power system. Fig.3 shows a single unit of the SBPC with every part already assembled. In order to save on system volume, low-level control functionality is also included such as, gate drive, isolated power supplies, current, voltage and condition monitoring.

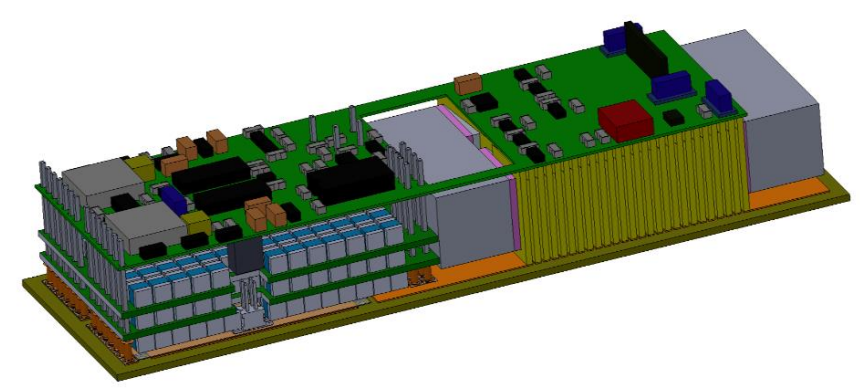

Fig. 3. Mechanical design of an SBPC unit.

As shown in Fig.3 the SBPC is composed of different levels of components. The power module is based on an Aluminium nitride substrate. On this are mounted the most energy dissipative components and associated peripherals, namely the SiC JFETs dies, P-MOSFETs, output inductors, current sensor, thermal sensor, power connections and main current paths. The internal PCBs are allocated to the input and output capacitors. Finally the top PCB is the interface unit which includes the gate drivers, current sensor transducer circuit and the thermal transducer circuit.

One of the main concerns with regard to the integration of passive components and increasing power density in general, is the thermal management system, since surfaces which are typically used to remove heat become smaller. In this case, the technique used to dissipate heat generated by the power devices is Jet Impingement cooling [5]. This technique is 
based on creating liquid cooling paths that direct the liquid cooling onto the hotspots of the substrate. The hotspots are created mainly by the SiC JFETS and the output inductor with residual cooling being provided to the P-MOSFETS. This highly efficient method of thermal management also gives the possibility of increasing the current density in the filter inductor to far greater levels (up to $50 \mathrm{~A} / \mathrm{mm}^{2}$ in the implementation described here) than is typically possible, allowing the energy density of the inductors to be increased.

Another important aspect which has been taken into consideration, during this development, is Electro-Magnetic Interference (EMI). Different techniques have been implemented in order to reduce the critical levels of EMI produced by the hard-switch of the semiconductors. The SBPC has been designed from the outset with EMI in mind and its internal structure is such that it contains the EMI produced. This is done by ensuring that there is a low impedance path to recirculate the common mode perturbations in the power module back to the originating disturbances. In addition the use of a PEEK plastic composite structure for the thermal management system eliminates the typical earthed connection to the heatsink. The elimination of earth connection and implementation of low impedance internal common mode paths helps to contain the EMI at source.

As mention above, the SBPC unit leads to a completely modular implementaion approach; self-contained and filtered output phase legs and can be paralleled in order to create either multi-phase or high power converters. Fig.4.a shows a three phase converter using three SBPC cells together with an input EMI filter. This could be arranged as a three leg inverter or a high-current, interleaved DC-DC converter for example.

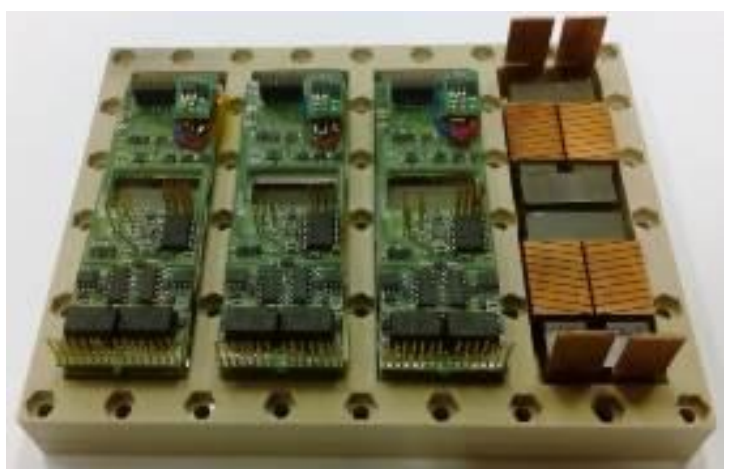

(a)

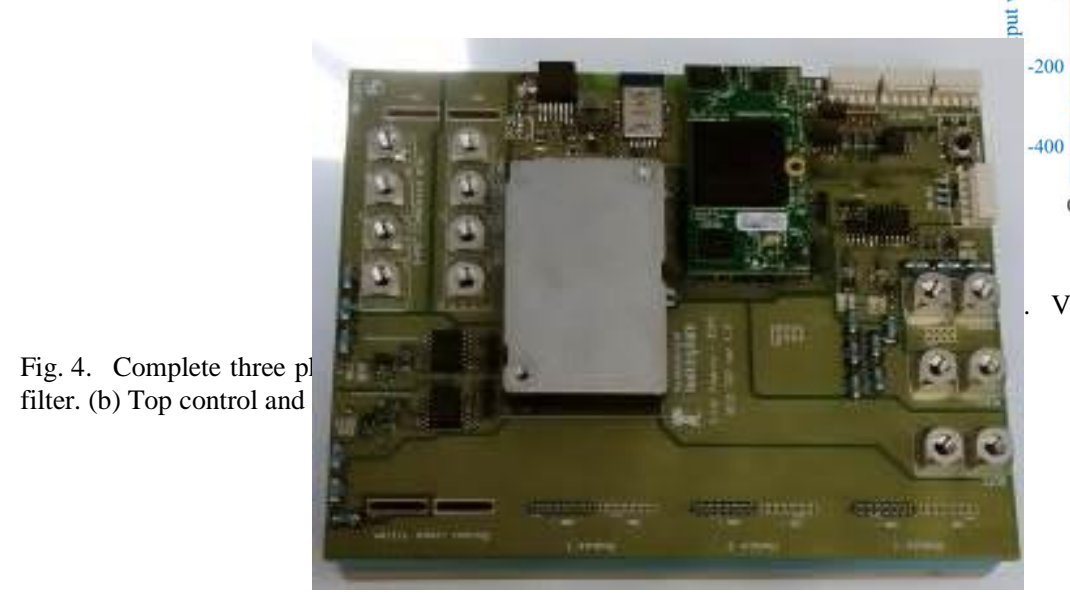

\section{EXPERIMENTAL RESULTS}

Fig. 5 shows the experimental voltage waveform at the midpoint of one of the half-bridge modules. Note the clean switching edges with no discernible overshoot and minimal ringing, highlighting the effective integration of the input capacitors and creation of the low inductance commutation loop. Fig.6 shows experimental waveforms taken from the power module when configured as an inverter. The load is purely resistive and it can be appreciated that the output voltage and the output current are completely sinusoidal with a low level of ripple. Typically, a standard VSI produces a switching waveform at its output, often there is a need to filter the high frequency harmonics where long feeder lines or stringent power quality requirements are in place. The SBPC includes those filters (values are $L_{o}=25 \mu \mathrm{H}$ and $C_{o}=1.8 \mu \mathrm{F}$ ). The advantages of including filters inside the power module are numerous: the power converter system can be made to be more compact, preventing propagation of harmonics and EMI, while from an electrical machine perspective, the levels of damaging high speed common mode voltage (CMV) are drastically reduced, increasing the electrical machine's lifetime.

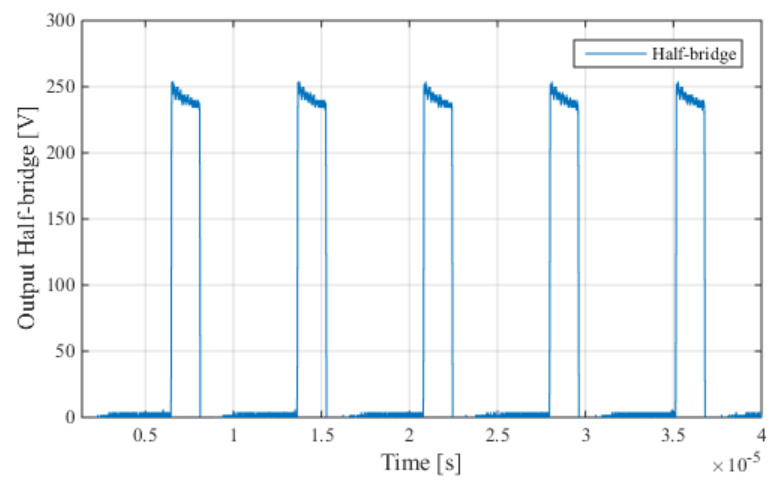

Fig. 5. Bridge mid-point voltage waveform.

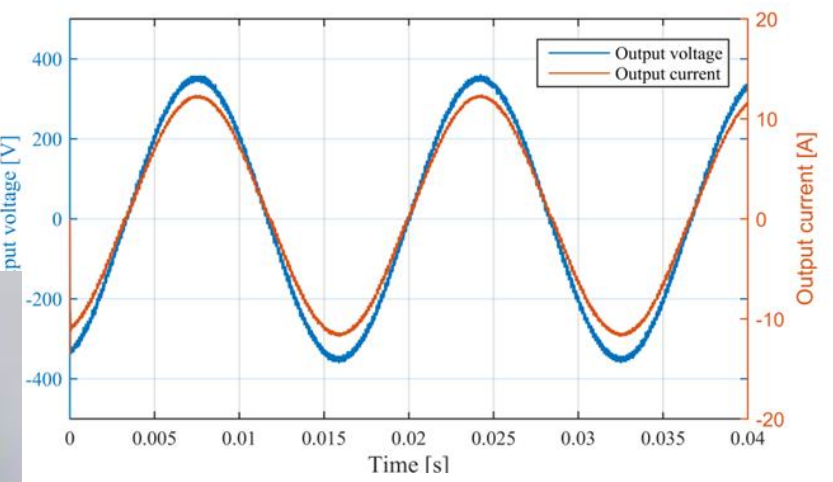

Voltage and current output of SBPC module. 


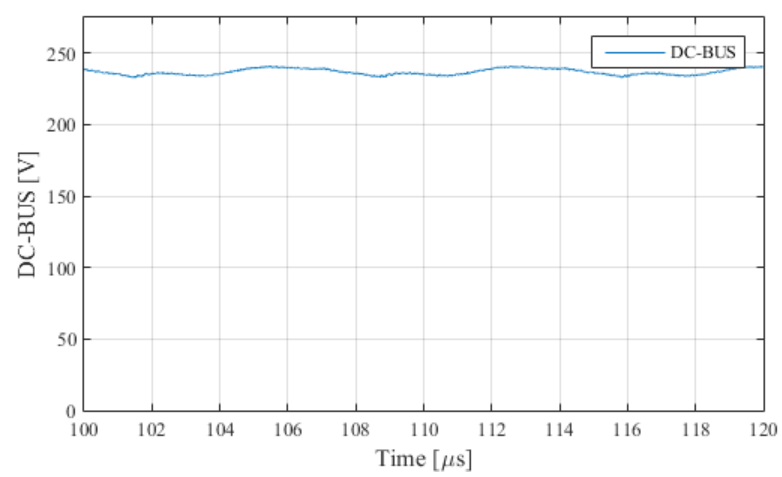

Fig. 7. DC-Bus voltage converter in DC/DC mode.

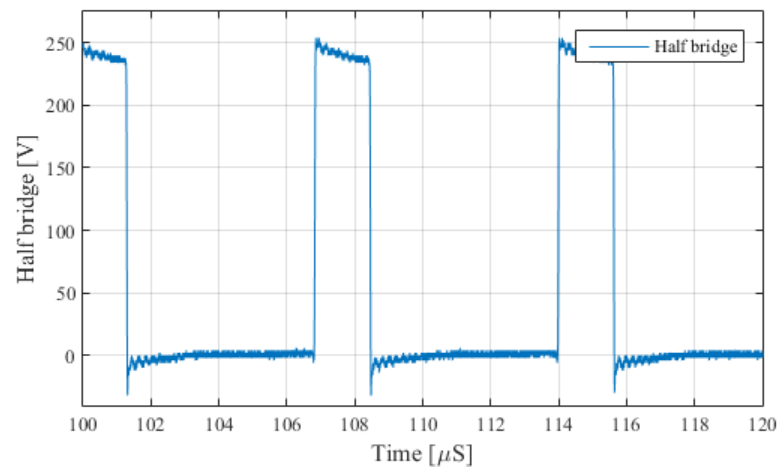

Fig. 8. Half bridge output voltage on DC/DC mode with $20 \%$ duty cycle.

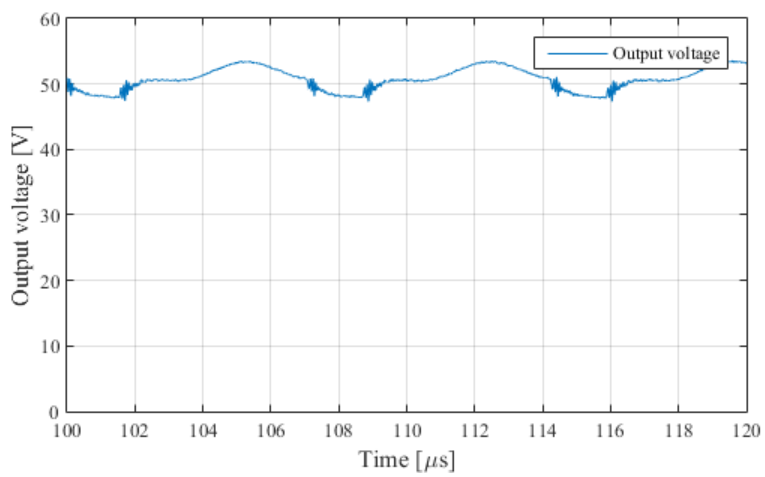

Fig. 9. Output voltage on DC/DC mode with fixed duty cycle.

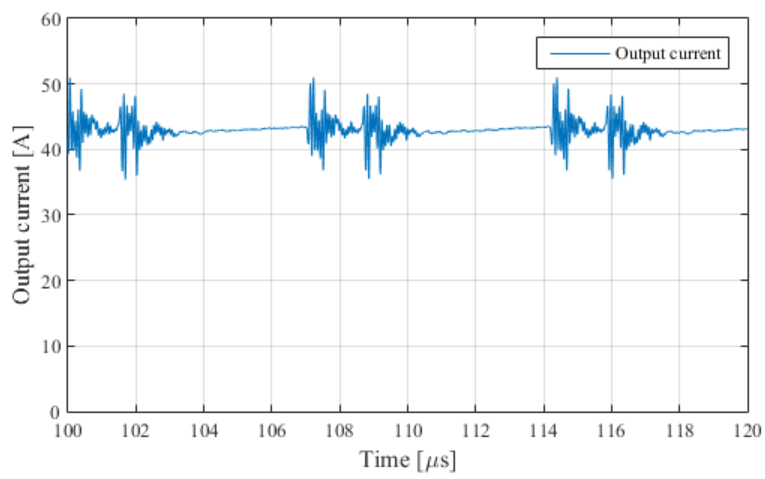

Fig. 10. Output current on DC/DC mode with fixed duty cycle.

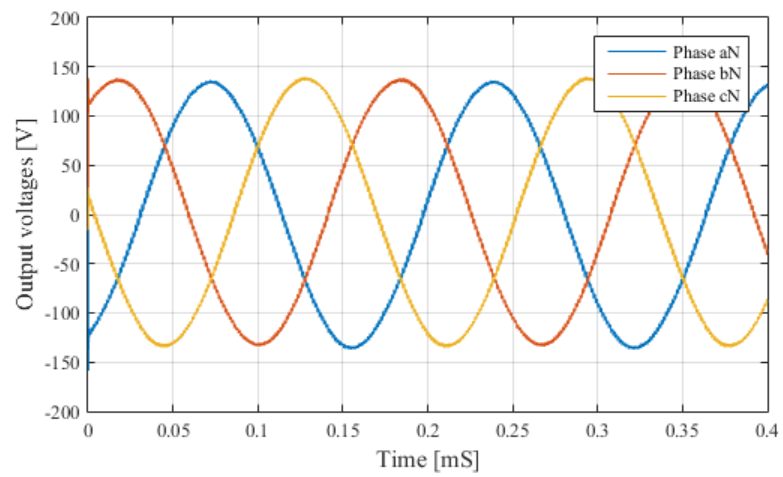

Fig. 11. Output voltages in three phase converter mode.

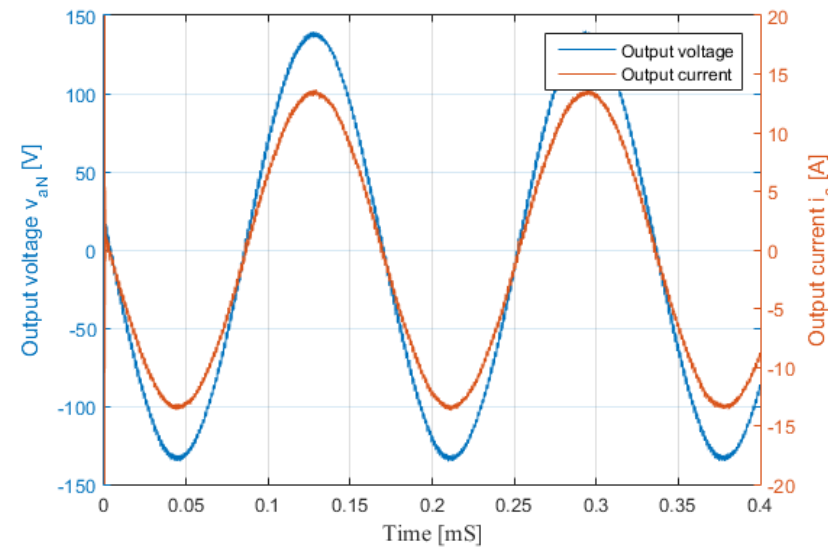

Fig. 12. Output phase voltage and current in three phase converter mode.

The power converter structure can also be used as a DC-DC converter since all passive components necessary in a typical DC-DC converter are integrated within each switching cell, Figs 7-10 show the operation of a single cell in a typical arrangement where the nominal input voltage of $240 \mathrm{~V}$ with an output voltage of $\sim 48 \mathrm{~V}$. The converter is driven with an open loop fixed $20 \%$ duty cycle, hence the poor regulation on the output voltage. Fig 7 shows the input DC voltage and the output switching node of the devices is shown in Fig 8. Fig 9 
shows the output voltage and Fig 10 shows the output current. Note probe induced noise is present on the output current waveforms and has not been pre-filtered for display purposes. Clearly, given enough switching cells, any number of output phases can be created from the modular structure, Figures 10 and 11 show results from the converter when three independent cells are used to create a three-phase inverter. It can be clearly seen that the quality of the output waveforms would negate the need for any form of $\mathrm{dv} / \mathrm{dt}$ filter for long line or sensitive motor use.

\section{CONCLUSIONS}

This paper presented a modular, power-cell solution which allows the creation of any two level topology converter. The cell structure enables fast switching of wide bandgap semiconductor devices while allowing high power converters to be fabricated using multiple, smaller commutation cells. An example 3-phase inverter module demonstrates that the level of integration and power density can be increased substantially without compromising converter performance. On the contrary, the techniques for integrating input and output filters enable dramatically reduced levels of EMI and minimal output ripple while realising converter power densities in excess of $50 \mathrm{~kW} /$ litre.

\section{REFERENCES}

[1] A. Nawawi, T. Chin Foong, L. Yitao, A. Sakanova, Y. Shan, L. Yong, et al., "Design of high power density converter for aircraft applications," in Electrical Systems for Aircraft, Railway, Ship Propulsion and Road Vehicles (ESARS), 2015 International Conference on, 2015, pp. 1-6.

[2] K. Koiwa and J. I. Itoh, "A Maximum Power Density Design Method for Nine Switches Matrix Converter Using SiC-MOSFET," Power Electronics, IEEE Transactions on, vol. 31, pp. 1189-1202, 2016.

[3] E. Levi, "Multiphase Electric Machines for VariableSpeed Applications," Industrial Electronics, IEEE Transactions on, vol. 55, pp. 1893-1909, 2008.

[4] R. Bojoi, A. Cavagnino, A. Tenconi, and S. Vaschetto, "Multiphase PM machine for More Electric Aircraft applications: Prototype for design validation," in IECON 2012 - 38th Annual Conference on IEEE Industrial Electronics Society, 2012, pp. 3628-3634.

[5] Skuriat, R.; Johnson, C.M., "Direct substrate cooling of power electronics," in Power Electronics and Applications, 2009. EPE '09. 13th European Conference on , vol., no., pp.1-10, 8-10 Sept. 2009 